\title{
Referral patterns, cancer diagnoses, and waiting times after introduction of two week wait rule for breast cancer: prospective cohort study
}

\author{
Shelley Potter, clinical fellow, ${ }^{1}$ Sasi Govindarajulu, staff grade breast surgeon, ${ }^{1}$ Mike Shere, associate \\ specialist breast physician, ${ }^{1}$ Fiona Braddon, clinical information systems manager, ${ }^{2}$ Geoffrey Curran, cancer \\ analyst, ${ }^{3}$ Rosemary Greenwood, medical statistician, ${ }^{4}$ Ajay K Sahu, consultant surgeon, ${ }^{1}$ \\ Simon J Cawthorn, consultant surgeon ${ }^{1}$
}

Breast Care Centre,

Frenchay Hospital, Bristol BS16 1JE

${ }^{2}$ Administration Building,

Frenchay Hospital

${ }^{3}$ Southmead Hospital, Bristol

${ }^{4}$ Research and Development Unit, United Bristol Healthcare Trust, Bristol

Correspondence to: S J Cawthorn Simon.Cawthorn@nbt.nhs.uk

doi:10.1136/bmj.39258.688553.55

\section{ABSTRACT}

Objective To investigate the long term impact of the two week wait rule for breast cancer on referral patterns, cancer diagnoses, and waiting times.

Design Prospective cohort study.

Setting A specialist breast clinic in a teaching hospital in Bristol.

Participants All patients referred to breast clinic from primary care between 1999 and 2005.

Main outcome measures Number, route, and outcome of referrals from primary care and waiting times for urgent and routine appointments.

Results The annual number of referrals increased by $9 \%$ over the seven years from 3499 in 1999 to 3821 in 2005. Routine referrals decreased by $24 \%$ (from 1748 to 1331), but two week wait referrals increased by $42 \%$ (from 1751 to 2490) during this time. The percentage of patients diagnosed with cancer in the two week wait group decreased from $12.8 \%(224 / 1751)$ in 1999 to $7.7 \%(191 / 2490)$ in 2005 ( $P<0.001)$, while the number of cancers detected in the "routine" group increased from $2.5 \%(43 / 1748)$ to $5.3 \%(70 / 1331)$ (P<0.001) over the same period. About $27 \%$ (70/261) of people with cancer are currently referred in the non-urgent group. Waiting times for routine referrals have increased with time. Conclusion The two week wait rule for breast cancer is failing patients. The number of cancers detected in the two week wait population is decreasing, and an unacceptable proportion is now being referred via the routine route. If breast cancer services are to be improved, the two week wait rule should be reviewed urgently.

\section{INTRODUCTION}

At the end of the 20th century, cancer services in the United Kingdom were in a state of disarray. Mortality for a range of malignancies, including breast cancer, was among the highest in Europe ${ }^{1}$ and "disgracefully" long waiting lists resulting in delayed diagnosis and treatment were thought to be at least partly responsible. ${ }^{2}$ In 1998, in an attempt to address these inequalities and to improve outcomes for patients with breast cancer, the Department of Health issued a circular titled Breast cancer waiting times - achieving the two week target. ${ }^{3}$ The aim of this circular and the later NHS Cancer Plan was to improve access to specialist services, thus facilitating early diagnosis and treatment. The resultant fast track or two week wait rule stipulated that, by April 1999, all patients with suspected breast cancer should be seen by a specialist within two weeks of referral by a general practitioner, and, despite having little scientific foundation, ${ }^{24-6}$ this "guarantee" conveyed a genuine sense of commitment to improving cancer services in the UK.

This initial optimism, however, was short lived. From the beginning, the value and effectiveness of the two week wait rule have been questioned. The number of cancers detected in this group of patients has been low, ${ }^{7}$ and the poor predictive value of fast track referral guidelines together with poor adherence in primary care has flooded one stop clinics with large numbers of inappropriate referrals. ${ }^{2}$ As a result, waiting times for those patients deemed non-urgent by the general practitioner have significantly increased, ${ }^{89}$ prompting considerable criticism because up to a third of cancers are ultimately diagnosed from this group. ${ }^{8}$ No survival benefit has been shown, ${ }^{7}$ and several authors have called for a re-evaluation of the system. ${ }^{4}$

For psychological and oncological reasons, all patients with suspected malignancy deserve to be seen promptly and treated effectively, regardless of their diagnosis. We analysed referrals from primary care to a specialist breast unit over a seven year period to evaluate the impact of the two week wait rule in our patients.

\section{METHODS}

The Frenchay Breast Care Centre serves a population of 500000 in Bristol and northeast Somerset and is one of two centres providing services for the Avon breast screening programme. Each year, we see over 4000 new patients and close to 400 new cases of cancer are diagnosed. The centre is staffed by two consultant surgeons, one associate specialist breast physician, one 


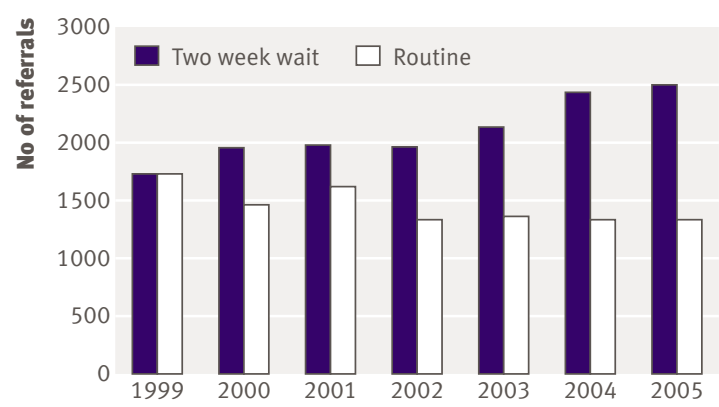

Year

Fig 1 Trends in referral patterns from primary care to breast clinic, 1999-2005

staff grade breast surgeon, one specialist registrar, two breast care nurses, and one part time follow-up clinic nurse.

We prospectively collected data on the number, route, and outcome of patients referred from primary care to the centre from 1999 to 2005 using standard data collection sheets that were completed at the time of consultation and diagnosis and subsequently used to generate outcome letters to primary care. This information was collated by using the hospital patient administration system. The referrals were classified by the referring general practitioner as being either "urgent" according the two week wait criteria (see box) or "routine." We compared the number of cancers detected and waiting times for clinic appointments in each group over time. We assessed the significance of any changes seen in referral patterns with Poisson regression analysis with a log link using Stata software and any changes in the percentages of cancers referred in each group with logistic regression.

\section{RESULTS}

Over the seven years, the centre received 24999 new referrals from primary care, a mean annual referral rate of 3571 (SD 182). From 1999 to 2005, the number of referrals increased by an apparent $9 \%(n=322)$ from

\section{Criteria for two week wait referral}

\section{Patient of any age with:}

A discrete hard lump with fixation, with or without skin tethering

Unilateral eczematous change in the skin or nipple that does not respond to topical treatment

Nipple distortion of recent onset

Spontaneous unilateral bloody discharge from nipple

Skin distortion, tethering, discolouration, nodule, or ulceration

New lump or suspicious features in patient with previous diagnosis of breast cancer

Female aged $>30$ years with:

Discrete lump persisting after period/menopause

Fixed, hard, or enlarging lump

Male aged $>50$ with:

A unilateral firm subareolar mass with or without nipple distortion or associated skin changes
3499 in 1999 to 3821 in 2005, a significant increase of $1.6 \%(95 \%$ confidence interval $1.0 \%$ to $2.2 \%)$ or about 56 additional new referrals a year.

As figure 1 shows, however, the number of two week wait referrals has dramatically changed, increasing by an apparent $42 \%(\mathrm{n}=739)$ from 1751 in 1999 to 2490 in 2005 , an estimated increase of $5.8 \%$ a year $(5.0 \%$ to $6.7 \%, \mathrm{P}=0.001)$. By contrast, the number of routine referrals has declined over the same period by an estimated $4.3 \%$ a year $(3.3 \%$ to $5.2 \%, \mathrm{P}<0.001)$, giving an apparent reduction of $24 \%(n=417)$ from 1999 to 2005 .

Despite the changes in referral patterns, the total number of cancers diagnosed in those referred from primary care has remained fairly constant over the seven years, with a mean of 263 (SD 18) new cancers diagnosed annually from this group (fig 2). Initially, in 1999 , most of these cancers presented in the two week wait group ( $\mathrm{n}=224,84 \%$ of all cancers referred by general practitioner, $12.8 \%$ of all urgent/two week wait referrals) with only a small number $(n=43,16 \%$ of all cancers referred by general practitioners, $2.5 \%$ of all routine referrals) referred as routine. With time, however, despite the increasing numbers of urgent two week wait referrals, the actual number of cancers diagnosed in this group has fallen. In 2005, only 191 out of 261 cancers $(73 \%$ of all cancers referred by general practitioners, $7.7 \%$ of all urgent referrals) were referred as two week waits while the number of cancers diagnosed in the routine group increased over the same period. The chances of being diagnosed with cancer in the two week wait group have decreased each year with an odds ratio of 0.91 (0.89 to 0.94 ), meaning patients in this group are 0.91 times as likely to be diagnosed with cancer in each subsequent year, which is highly significant $(\mathrm{P}<0.001)$. In 2005, 70 out of 261 cancers $(27 \%$ of all cancers referred via the general practitioner, $5.3 \%$ of all routine referrals) were referred as non-urgent. This constitutes an increase in the chances of being diagnosed with cancer in the routine group with an odds ratio of 1.21 (1.14 to 1.28$)$; thus

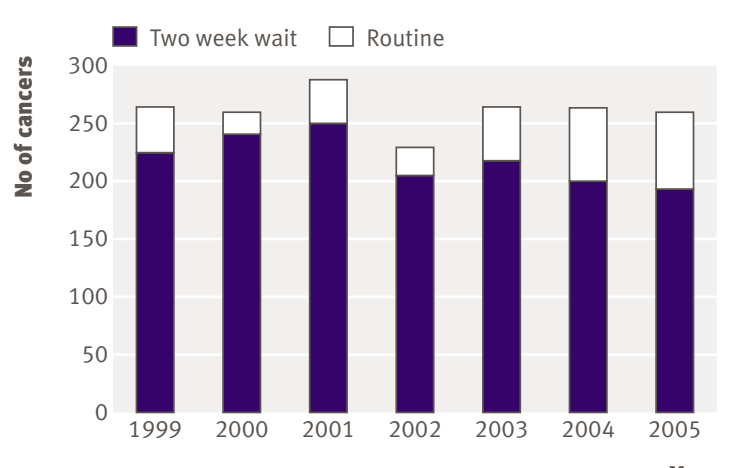

Fig 2 | Cancers diagnosed in patients referred from primary care, 1999-2005. Overall number of cancers referred has remained constant. The number of cancers diagnosed from the two week wait group has decreased with time, despite increasing numbers of referrals, while number of cancers referred as "routine" has increased over same period 


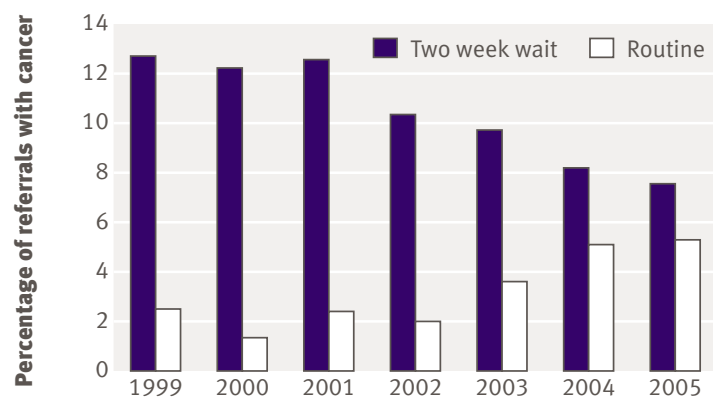

Year

Fig 3 | Percentage of referrals with diagnosis of cancer by referral group. Percentage of two week wait referrals diagnosed with cancer has declined despite increasing numbers of referrals. Reverse is true of routine referrals, suggesting decreased diagnostic accuracy in primary care

patients are 1.21 times more likely to be diagnosed with cancer in the routine group in each subsequent year, which is, again, a highly significant change $(\mathrm{P}<0.001)$. These trends are summarised in figure 3 and the table.

Despite increasing numbers of referrals, waiting times in the two week wait group have always been maintained well within the target range, but, as predicted, this increasing demand on services has affected non-urgent patients and the waiting times for routine referrals have increased. The decrease in waiting times from 2000 to 2002 seen in figure 4 reflects an increase in clinic capacity created by the introduction of a new follow-up policy. After 2003, however, waiting times have steadily increased, reflecting the increasing numbers of patients referred under the two week wait. The current waiting time for a routine referral is 30 days.

We also analysed the demographics of the population served by the centre and the general practitioners referring to breast clinics from 1999 to 2005 . Over this time, the population of general practitioners referring to the clinic did not change significantly, nor did the demographics of the population served by the hospital.

\section{DISCUSSION}

The two week wait rule was supposed to improve access to specialist services for all patients with suspected breast cancer and to facilitate early diagnosis and treatment. This policy has failed to achieve its goal. Since the introduction of the rule, while the number of patients referred under the urgent or two week wait rule has steadily increased by about $5.8 \%$ a year from 1751 in 1999 to 2490 in 2005, the percentage of these patients ultimately diagnosed with cancer has significantly decreased from $12.8 \%$ in 1999 to $7.7 \%$ in 2005. More worryingly, however, despite a significant decrease in number of routine referrals (1748 in 1999 to 1331 in 2005), the proportion of cancers diagnosed in this group has significantly increased $(2.5 \%$ in 1999 to $5.3 \%$ in 2005$)$ over the same period. These patients are also potentially being disadvantaged by longer clinic waits and delays in diagnosis as waiting times for routine referrals have increased in the face of increasing service demands from the dramatically increased number of patients referred under the two week wait rule, over $90 \%$ of whom have benign disease.

\section{Strengths and weaknesses}

We evaluated a large cohort of patients over a prolonged period and assessed the resultant trends. We used robust diagnostic and outcome data recorded from forms subsequently used to generate letters to primary care on almost 25000 referrals over the seven years. Our results are therefore an accurate reflection of the impact of the policy on referrals and diagnoses of cancer in our centre. Though we evaluated referrals to a single centre only, because of the size of the study population and the workload of the clinic we consider that these findings could be extrapolated to other centres and probably reflect the referral patterns and rates of cancer diagnosis seen by other specialist breast units nationwide.

\section{Comparison with other studies}

Many studies have questioned the validity of the two week wait rule, particularly with respect to the low number of cancers detected ${ }^{7}$ in this group, and have discussed the unacceptability of what is effectively a two tier system, whereby so called "non-urgent referrals" have to wait longer to see a specialist. ${ }^{5}$ Concerns are justified given the fact that a large proportion of cancers detected are in patients referred by this route. $^{8}$ In our study, more than one in four $(27 \%)$ patients ultimately diagnosed with cancer in 2005 was referred non-urgently, which is consistent with earlier findings of between $20 \%$ and $36 \%{ }^{811}$ Many of these studies, however, were conducted soon after the introduction of the policy and offer no information regarding the long term impact of the two week wait rule or how this has changed with time. With the exception of a study of 15 breast units conducted by Sauven, ${ }^{8}$ which evaluated over 12000 referrals, many of these early studies were also small. ${ }^{912}$ Our study is based on robust

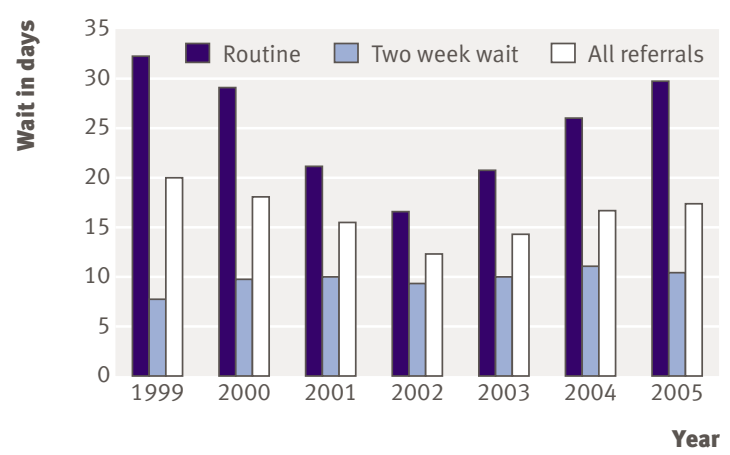

Fig 4 | Waiting times for appointments. Increasing numbers of two week waits have affected clinic capacity, increasing waiting times for routine referrals. Decrease in waiting times for routine referrals seen from 2000 to 2002 was caused by increased clinic capacity created by change in follow-up policy 


\section{WHAT IS ALREADY KNOWN ON THIS TOPIC}

The two week wait for patients with suspected breast cancer was introduced in 1999 to improve outcomes by improving access to specialist services and facilitating prompt diagnosis and treatment

Its effectiveness has been questioned because of the low number of cancers detected in the two week referral group and poor prognostic value of the referral guidelines

\section{WHAT THIS STUDY ADDS}

Over a seven year period, the number of two week referrals has dramatically increased, but the percentage of cancers detected in this group has decreased, and the number of routine referrals has decreased, but more cancers are now detected in this group

The two week wait rule has led to increased waiting times for routine appointments

The two week wait rule is failing to meet its aims

prospective data collected from 25000 referrals over seven years and assesses the long term impact of the two week wait rule policy on numbers of referrals and cancer diagnoses in a specialist setting. It extends previous findings and shows several worrying trends, which add further weight to the debate over the two week wait rule.

Why is the two week wait rule failing?

In 2005 , only $7.7 \%$ of all two week wait referrals were ultimately diagnosed with cancer compared with $12.8 \%$ of urgent referrals when the two week wait was first introduced. This is compared with the alarming increase in cancers diagnosed from the "routine" population, from $2.5 \%(n=43)$ in 1999 to $5.3 \%(n=70)$ in 2005. These data support other studies in suggesting that the diagnostic accuracy of general practitioners has decreased since the introduction of the two week wait. ${ }^{9}$ It has been suggested that general practitioners might not suspect cancer in many patients presenting to them with malignancy. ${ }^{13}$ It is more likely, however, that this apparent decrease in "diagnostic accuracy" is the result of the poor predictive value of the two week referral criteria rather than a decline in general practitioners' diagnostic skills. The guidelines are less discerning than traditional clinical judgment and general practitioners' skills are being undermined by the need to adhere to the two week wait guidelines. General practitioners themselves have expressed concerns about the system and clinical uncertainty, often overreferring as a result of media pressure and patients' expectations. ${ }^{14}$ Indeed, a recent study showed identical presenting symptoms in patients diagnosed with breast cancer regardless of their route of referral. ${ }^{15}$

Waiting times for routine referrals have also increased as clinic capacity has been diverted to meet increasing demands from the two week wait population. The current waiting time for a routine appointment is 30 days, but in some centres may be significantly longer. ${ }^{8}$ This has considerable psychological impact for any patient, regardless of their diagnosis, ${ }^{16}$ but for the one in four patients with cancer in this group, the additional wait constitutes yet another unacceptable delay for definitive diagnosis and treatment. The system is failing patients and a change is urgently needed.

Contributors: SP performed the literature search, collected and analysed the data, and wrote the paper. SG, MS, and AKS were involved in data collection and writing. FB and GC were involved in data collation and analysis. Helen Cooke analysed the demographics of the hospital catchment area to ensure this has remained stable. $\mathrm{RG}$ performed the statistical analysis. SJC was responsible for the design and conception of the study, assisted in writing, and is guarantor. Funding: None.

Competing interests: None declared.

Ethical approval: Not required.

1 Bray F, Sankila R, Ferlay J, Parkin DM. Estimates of cancer incidence and mortality in Europe. Eur J Cancer 2002;38:99-166.

2 Jones R, Rubin G, Hungin P. Is the two week rule for cancer referrals working? BMJ 2001;322:1555-6.

3 Health Service Circular HCS 1998/242. Breast cancer waiting times -achieving the two week target. 1998. www.dh.gov.uk/ PublicationsAndStatistics/LettersAndCirculars/ HealthServiceCirculars/HealthServiceCircularsArticle/fs/en? CONTENT_ID=4004290\&chk=Mvye/Y

4 Fentiman IS. Two week wait for suspected cancer: milestone or millstone? Int J Clin Pract 2005;59:1247-52.

5 Cant PJ, Yu DSL. Impact of the "2 week wait" directive for suspected cancer on service provision in a symptomatic breast clinic. Br J Surg 2000;87:1082-6.

6 Coates AS. Breast cancer: delays, dilemmas and delusions. Lancet 1999;353:1112-3.

7 Hanna SJ, Muneer A, Khalil KH. The 2-week wait for suspected cancer: time for a rethink? Int J Clin Pract 2005;59:1334-9.

8 Sauven P. Impact of the " 2 week wait" on referrals to breast units in the UK. Breast 2002;11:262-4.

9 Khawaja AR, Allan SM. Has the breast cancer "two week wait" guarantee for assessment made any difference? Eur J Surg Oncol 2000;26:536-9.

10 National Institute of Clinical Excellence. NICE guideline-referral for suspected cancer. June 2005. www.nice.org.uk/CG027

11 Paice A, Ooo M, Shah E. The two week wait-are breast cancers being missed? Eur J Surg Oncol 2006;32:A1036.

12 Patel RS, Smith DC, Reid I. One stop breast clinics-victims of their own success. A prospective audit of referrals to a specialist breast clinic. Eur J Surg Oncol 2000;26:452-4.

13 Sauven P. Specialists, not GPs may be best qualified to assess urgency. BMJ 2001;323:864-5.

14 Dodds W, Morgan M, Wolfe C, Raju KS. Implementing the 2-week rule for cancer referral in the UK: general practitioners' views and practices. Eur J Cancer Care 2004;13:82-7.

15 Griffiths C, Raine C, Sauven P, Mason S. Patients with breast cance present with identical symptoms in the urgent 2 week wait stream and the routine stream. Eur J Surg Oncol 2006;32:1042.

16 Ganz PA. Quality of life across the continuum of breast cancer care. Breast J 2000;6:324-30.

Accepted: 14 June 2007 